\title{
Interference effect on friction behavior of asperities on single crystal copper
}

\author{
Xiaoming Liu ${ }^{\mathrm{a}, *}$, Bin Zhang ${ }^{\mathrm{b}}$, Yueguang $\mathrm{Wei}^{\mathrm{a}}$ \\ a LNM, Institute of Mechanics, Chinese Academy of Sciences, 100190 Beijing, China \\ ${ }^{\mathrm{b}}$ State Key Laboratory of Mechanics and Control of Mechanical Structures, College of Aerospace Engineering, Nanjing University of Aeronautics and \\ Astronautics, 210016 Nanjing, China
}

\section{A R T I C L E I N F O}

Article history:

Received 15 July 2014

Received in revised form

8 August 2014

Accepted 16 August 2014

Available online 2 September 2014

Keywords:

Friction transition

Plastic behavior

Green function molecular dynamics

Asperity interference

\begin{abstract}
A B S T R A C T
By using Green function molecular dynamics method, we systematically study the friction behavior of a single asperity and asperity array over the (1 1111$)$ surface of single crystal copper. We find that internal plastic behavior (burst of stacking faults, dislocation emission and propagation) is a promising reason for the higher value of static friction coefficient than that of dynamics friction in non-adhesive scratch. For the rough surface, however, the difference between static and dynamic friction coefficients disappear due to the interference between asperities. The interference dramatically increases the friction coefficient by introducing atomic scale plastic features (pile-up atoms, stacking faults, and U-shape dislocation loop).
\end{abstract}

(c) 2014 Elsevier Ltd. All rights reserved.

\section{Introduction}

Friction and wear at nano-size contacts are not the same as those in macroscale, since surface and interfacial phenomena becomes dominant in nanoscale. Measurements of nano-tribology properties (such as the friction coefficient, wear rate) depend highly on the surface structure, elastic/plastic/creep properties of material, and physical and chemical environments. The single asperity apparatus has been developed for providing mechanical properties of the surface with characteristic size from a few hundred nanometers to several microns. Experiments with single asperity apparatus such as the scanning force microscope (SFM) and atomic force microscope (AFM), are widely used in investigating friction behavior at nanoscale.

Continuum theories (Hertz, JKR [1], and DMT [2]) have been successfully used to explain adhesive or non-adhesive single asperity contact and friction. However, these theories aren't always precise. The study [3] by Mo et al. demonstrated the breakdown of continuum mechanics at nano-scale, which is explained by the rough (multi-asperity) nature of the contact. They showed that roughness theories of friction should be applied at the nanoscale. Further study [4] showed that roughness theories captured the correct physics of deformation at the nanoscale, and the number of atoms interacting across the interface actually dominated the

\footnotetext{
* Corresponding author.

E-mail addresses: xiaomingliu@Inm.imech.ac.cn (X. Liu), beenchang@nuaa.edu.cn (B. Zhang), ywei@imech.ac.cn (Y. Wei).

nano-friction behavior. Experiment [5] observed that the size of nanoscale asperities distributed on the AFM diamond tip is in the order of few nanometers. The nanoscale roughness can be represented by an array of asperities in multi-scale models [6] which extract the force-displacement data of a single asperity from molecular simulations. Pile-up of nano-scale material plays a similar important role as the nanoscale roughness factor, Mishra et al. [7,8] developed an analytical model to study plowing friction for the single-asperity contact at nanoscale, their results proved that the material pile-up has a large contribution to nano-friction. Local roughness may affect frictional strength by multi-scale nature $[9,10]$ or flattening characteristics [11], its effect on dislocation pattern has been studied during nano-scale metallic contacts [12,13], but has not been presented for nano-scratch process.

Another interesting topic has been discussing for decades: Why is the coefficient of static friction normally higher than the value of dynamic friction? The reasons which have been discovered include several factors as following: The adhesive interaction between the surfaces is well established when the surfaces are at rest, the relative motion gives less time for those stronger bonds to form; The inertia effect is one factor, the applied force has to overcome both friction and the inertia for the start of sliding in the case of static friction; The surface roughness is another factor, irregularities between surfaces make the fitting of two surfaces by peaks and valleys, thus a larger applied force is needed for starting the body's motion. While the surfaces are moving, all asperities are hopping along, skipping along the surface; The third body explanation is that elastic particles tend to deform and lock themselves together, thus the two sliders form a 
kind of ratcheting effect when there is no relative movement. In this work, we study the effect of internal plastic behavior on the transition from static to dynamic friction. The obvious reason for this factor is that plastic behaviors (stacking fault burst, dislocation nucleation and propagation) are all rate dependent, and are very sensitive to the local instability caused during friction contact. And also, discrete behavior of dislocation is much more critical in small scale deformation than macro scale $[14,15]$. But to authors' knowledge, no study has ever discussed about the contribution of internal friction of material on this topic, the contribution can be more critical during the nano-scratch process since the discrete effect plays a more important role.

In our study, we use Green function molecular dynamics (GFMD) method to study the effect of internal plastic behaviors (stacking fault, dislocation emission and propagation) on nanofriction behavior, its contribution for the higher value of static friction coefficient is discussed. The interference effect between asperities is also studied for understanding roughness effect.

\section{Method}

Studying indentation or scratch using molecular dynamics normally requires very high computational cost. However, the cost can be largely reduced. Because according to Hertz contact theory [1], the deformation field decays with the increase of the distance from the contact point, with a $1 / r$ law. Thus full scale computation is not necessary because plastic deformation only appears near the scratch surface. The GFMD can be used to take account of the longrange interaction from atoms in the area far from the contact points. The computational cost can be reduced by replacing atoms in those areas with GFMD. This method has been effectively applied as a seamless boundary condition for cases where near-surface deformation are dominated, such as surface relaxation [16], contact [17], or calculation of surface phonon dispersion [18].

We use the method developed by Campaná and Müser [19], and further implemented by Kong et al. [17], the scheme of the method is shown in Fig. 1, which is divided to two steps. First, the effective elastic stiffness coefficients of the GFMD atoms, from the bottom block in Fig. 1, are calculated by using the fluctuationdissipation theorem. In another word, the layer of GFMD atoms is connected with a layer of nonlinear springs, with stiffness matrix provided by using Green function method. Simulation of 500,000 time steps (time step of $1 f s$ ) is used to obtain the Green function matrix (elastic stiffness) in the reciprocal space. Second, in the scratch simulation, a single layer of the GFMD atoms are set at the bottom of the $z$ direction, which reproduces the effective elastic force from the bulk material by using the Green function matrix in the reciprocal space. This method significantly reduces the computational cost for indentation and scratch test, by considering only atoms near the surface.

During indentation or scratch simulation, boundary conditions are applied as follows. The top asperities are fixed, while one layer of atoms (set as Green function atoms) at the bottom is uniformly applied with normal force $\left(F_{n}\right)$ and horizontal force $\left(F_{t}\right)$. The force boundary conditions are similar to literature [20]. First, the normal force is applied piecewise in $300 \mathrm{ps}$ (time step $1 \mathrm{fs}$ ). Then the whole system is relaxed under NVT for a period of 100 ps before scratch. Simulations under various normal forces are conducted, and it has been verified that the system is fully relaxed before scratch that the indentation rate has no effect on the friction behavior.

In the simulation, we use force boundaries instead of velocity control [21]. The disadvantage of velocity control is that it leads to the inaccuracy of measuring the initial sliding force (static friction force). Theoretically, the acceleration is infinity once the velocity jumped from zero to the applied value at the initiation stage of sliding. The initial acceleration will cause an artificial contribution on the static friction force (the initial force required for sliding). Thus, in friction simulation, we gradually apply the friction force with a constant rate $0.03 \mathrm{nN} / \mathrm{ps}$ while keep $F_{n}$ as a constant value. The time evolution of the sliding distance, measured as the distance between the asperities and bottom slabs, is recorded. In order to eliminate thermal effect on the plastic behavior during scratch, the whole system is kept at a temperature of $10 \mathrm{~K}$ via an external NoseHoover thermostat. It should be noted that, the Green function matrix is also calculated at $10 \mathrm{~K}$. The scratch speed is not a constant but is below $150 \mathrm{~m} / \mathrm{s}$ during the scratch distance of $40 \mathrm{~nm}$, which is in the range normally used in MD simulations [22,23].

The simulation model consists of a crystal block at the bottom and a $2 \times 2$ asperity array (or just one asperity in single asperity test) at the top (see in Fig. 2). The bottom block is a single crystal copper with orientations as: $x-\left\langle\begin{array}{lll}1 & 1 & \overline{2}\rangle\end{array}, y-\langle\overline{1} 10\rangle\right.$ and $z-\left\langle\begin{array}{lll}1 & 1 & 1\end{array}\right\rangle$. The $x$ and $y$ directions are periodic, while top of $z$ direction is treated as the surface for scratch, and the bottom side of $z$ direction has atoms with Green function boundary condition applied. The dimension of the substrate in $x$ and $y$ direction is approximately $64 \mathrm{~nm}$ and $33 \mathrm{~nm}$, respectively, while the dimension in $z$ direction has different values in present study in order to check the dimension sensitivity in $z$ direction. The asperity array is composed of 4 rigid spheres with a square pattern as shown in Fig. 2, the alignment of the array is measured quantitatively by the rotation angle $\theta$. Our main concern is to investigate the

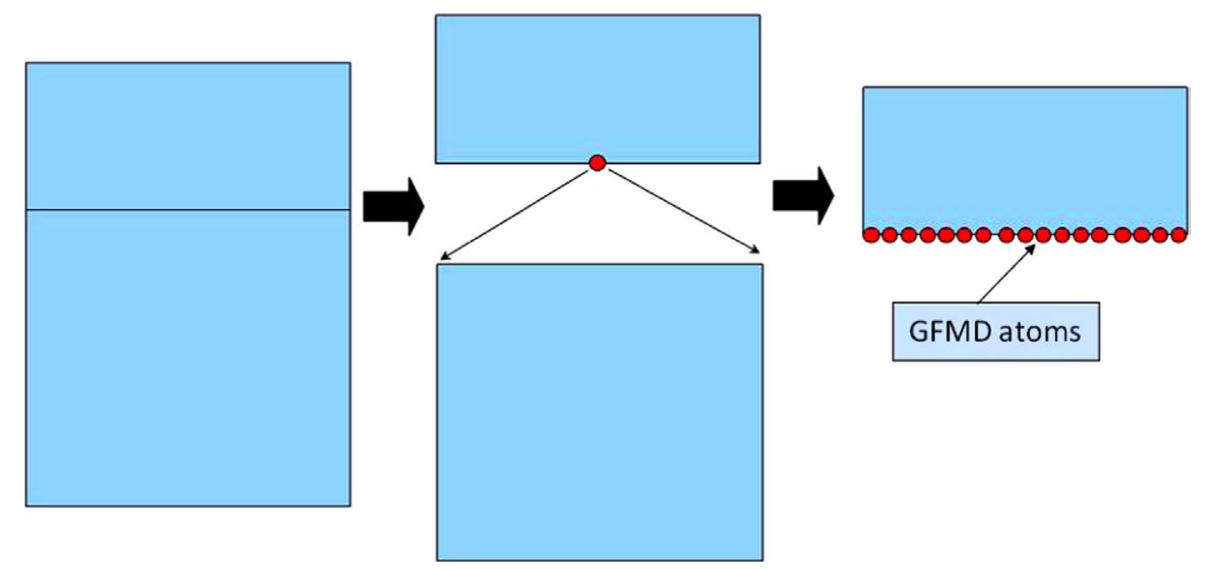

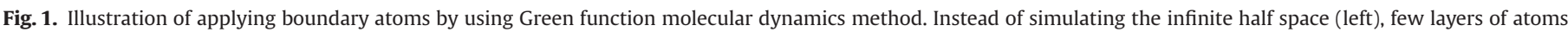

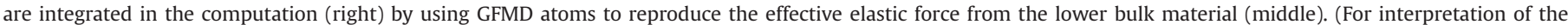
references to color in this figure legend, the reader is referred to the web version of this article.) 

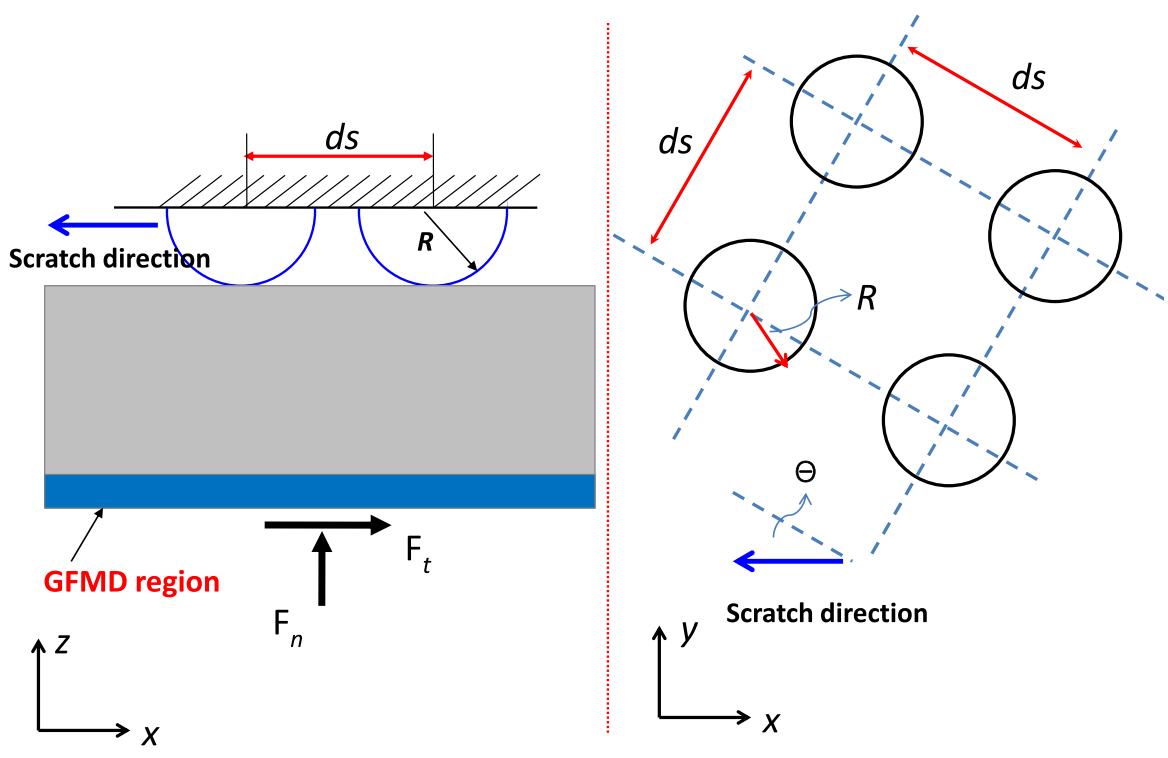

Fig. 2. Sketch of asperity array (left: $z-x$ view; right: $x-y$ view with rotation angle $\theta$, simulations with $\theta=0^{\circ}, 15^{\circ}, 30^{\circ}, 45^{\circ}$ are carried out.).

interference effect on the friction of multiple-asperities, the interdistance $d s$ is varied in the simulation to study the interference effect. The anisotropic effect on the friction by the arrangement of asperities is averaged out in this study as followed: The friction curve for asperity array with each set of $d s$ is averaged by using four cases with $\theta$ equal to $0^{\circ}, 15^{\circ}, 30^{\circ}$ and $45^{\circ}$, respectively. The symmetric feature of the (1 111 ) surface, as well as the asperity pattern are both taken into account by selecting the proper angles for realization. The interaction between the tip and the substrate is simulated by a repulsive potential as in Eq. (1):

$V= \begin{cases}A(R-r)^{3} & r \leq R \\ 0 & r>R\end{cases}$

where $A$ is the specified force constant (set as $530 \mathrm{nN} / \mathrm{nm}^{2}$ ) [24], $r$ is the distance from the atom to the center point of the rigid sphere, and $R$ is the radius of the indenter (set as $4 \mathrm{~nm}$ ) [24,25].

In the present study, we focus on studying the friction behavior with non-adhesive contact cases, which typically appears on passivated surfaces. Thus, the adhesion force is not considered in this study, we believe its effect on friction is profound and should be considered in the future study. Similar to work [26], we also did a verification test of indentation use a rigid diamond tip consisted with discrete carbon atoms, with Morse potential defined between the tip and the substrate, we define a cut off distance on the potential so that only repulsive part is taken into account. The verification shows that the difference on the force-displacement curve of indentation is very small. And also, plenty of studies also used present repulsive potential to study the indentation [27-29] or scratch test [30], and provide reasonable results. Thus, this potential has been proven to be effective for simulating on surfaces with low interface energy, e.g. passivated surfaces.

\section{Result and discussion}

\subsection{Model verification}

In this section, verification of the model is first presented, and then the realization method and the simulation parameters are discussed.
The friction curves of nano-scratch always fluctuate, this feature leads to the difficulty of quantitative analysis and comparison. The large fluctuation stems from the high vibration of atoms during scratch, also it may attribute from significant energy dissipation during friction [31], as indicated from various experiment curves [8]. Thus it needs average for rational comparison [11]. Fig. 3a shows the average friction curve with normal force $\left(F_{n}=180 \mathrm{nN}\right)$, the friction curve is not converged by averaging over five cases, but as shown in the figure, the difference between realizations of ten and twenty is pretty small, thus it can be concluded the simulation curve is converged by averaging over ten cases. In the following simulation, all the results are averaged over at least ten cases.

Within high resolution experiments at nano-scale [32-35], the indenter tip size is in the range of few nanometers, so that the normal force (contact force) of the tip is always in the range of one hundred nano-Newton [36] in order to prevent extra high stress during contact. Thus in this study, normal force $F_{n}$ at each asperity is selected from $0.8 f$ to $1.2 f$ (where $f=180 \mathrm{nN}$ ). The dimension of the system is selected as below: The size of the substrate in the $x$ (scratch direction) and $y$ direction is fixed as $64 \mathrm{~nm}$ and $33 \mathrm{~nm}$, respectively. The size of $z$ dimension (indentation direction) is chosen by using the advantage of GFMD so that the computation cost can be largely reduced. The dimension of $z$ direction (indentation direction) is verified as shown in Fig. 3b, it can be seen that the difference of friction curve is trivial between the selections of 30,42 and 54 layer of atoms. In the following simulations, 42 layers of atoms are chosen in the $z$ direction.

\subsection{Transition from static friction to dynamic friction for single asperity scratch}

For single asperity scratch, friction variation along the scratch is recorded in Fig. 4a, higher normal force leads to higher friction coefficient. All the curves have a rather high initial value followed by an abrupt decrease, and finally reach a stable regime. The initial resistance to sliding is considered as the static friction coefficient, and it is shown as a function of the normal load in Fig. 4b. The dynamic friction coefficient is taken as an average over the stable 
a

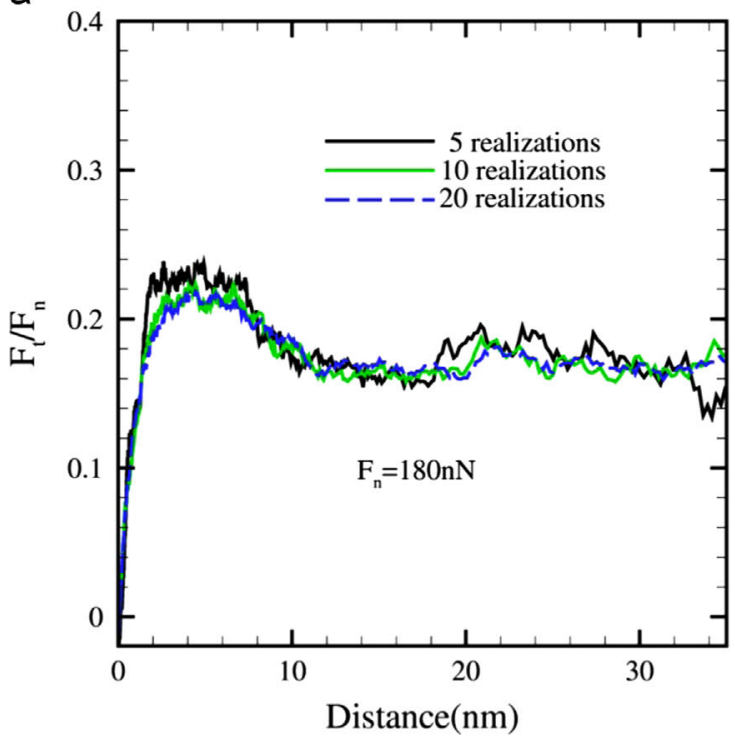

b

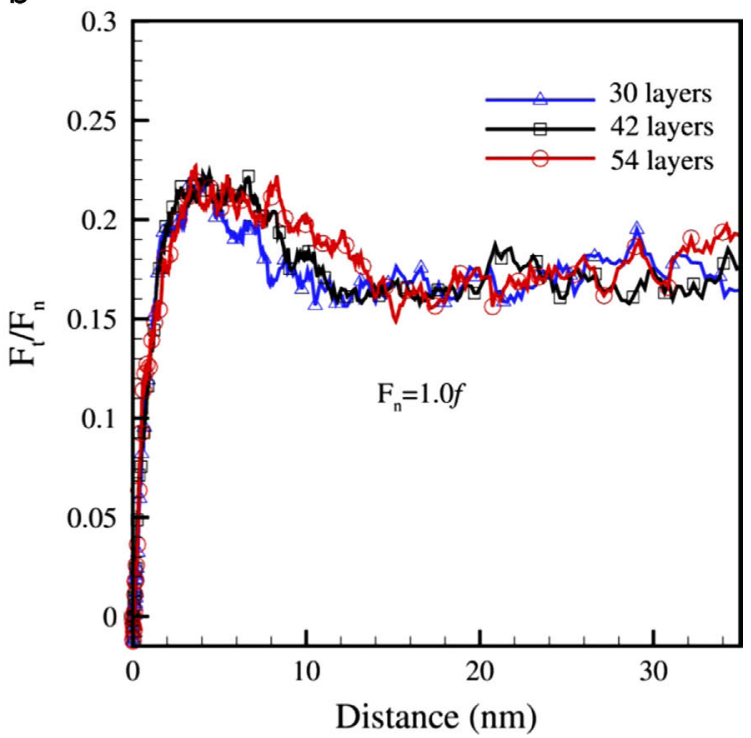

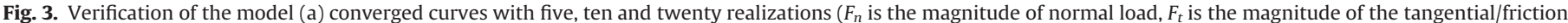
force.) (b) sensitivity of dimension size in the $z$ direction (all the friction curves are averaged over ten cases).

a

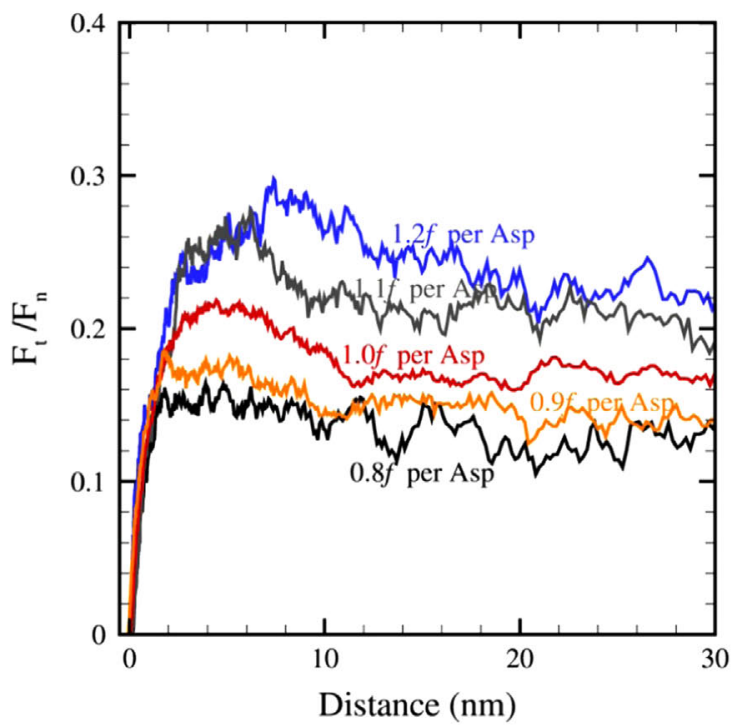

b

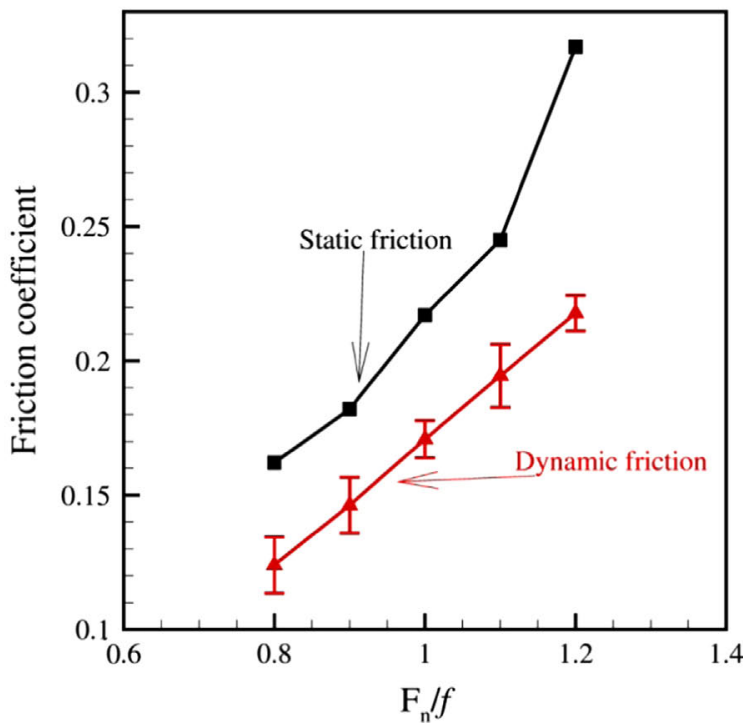

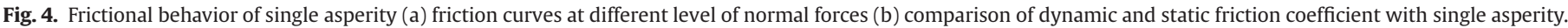
The error bar denotes the standard deviation over the scratch distance from $25 \mathrm{~nm}$ to $35 \mathrm{~nm}$.

regime (scratch distance from $25 \mathrm{~nm}$ to $35 \mathrm{~nm}$ ), also is shown in Fig. 4b. The static friction coefficient $\left(\mu_{s}\right)$ is higher than dynamic friction coefficient $\left(\mu_{d}\right)$ at different level of $F_{n}$. For all the situations, $\mu_{s}$ is at least $25 \%$ higher than $\mu_{d}$. The difference of friction coefficient is lowest for $F_{n}=0.8 f(\Delta \mu=0.039)$, and it monotonically increases with normal force $F_{n}$, it is highest for $F_{n}=1.2 f(\Delta \mu=0.1)$.

As suggested by work $[37,38]$, the chemical polishing and material removal make larger contributions to the friction and wear as the adhesive force is taken into account, thus the atoms are removed layer by layer during scratch. In our case, we study the friction while the interface energy is negligible, and in this situation, dislocation plasticity plays a more important role during scratch so that dislocation behaviors are the key attentions in our study. Discussions about the microstructure evolution during scratch are as follows.
In order to study the transition between $\mu_{s}$ and $\mu_{d}$, microstructure evolution during scratch is observed and recorded. Figs. 5 and 6 show the microstructure evolution during the scratch for $F_{n}=1.0 \mathrm{f}$ and $F_{n}=1.2 f$, respectively. It can be seen that stacking faults are activated on different slip planes between the initiation and the stable stage of sliding. At the initiation stage, the stacking faults are nucleated from slip planes $(\overline{1} 1 \overline{1}),\left(\begin{array}{lll}\overline{1} & \overline{1} & 1\end{array}\right)$ and $\left(\begin{array}{lll}1 & \overline{1} & 1\end{array}\right)$, see in top two figures of Fig. 5a and $b$ and Fig. $6 a$ and $b$ (the current positions are marked with hollow triangles, and the initial positions are marked with solid triangles). While during stable sliding, stacking faults in slip plane (1 11 ), which is parallel to the scratch surface, are generated, as marked by white circles in bottom two figures of Fig. 5b and Fig. 6b.

Schmid factors of 12 slip systems for normal and shear load are shown in Table 1. During indentation, Schmid factors from the 


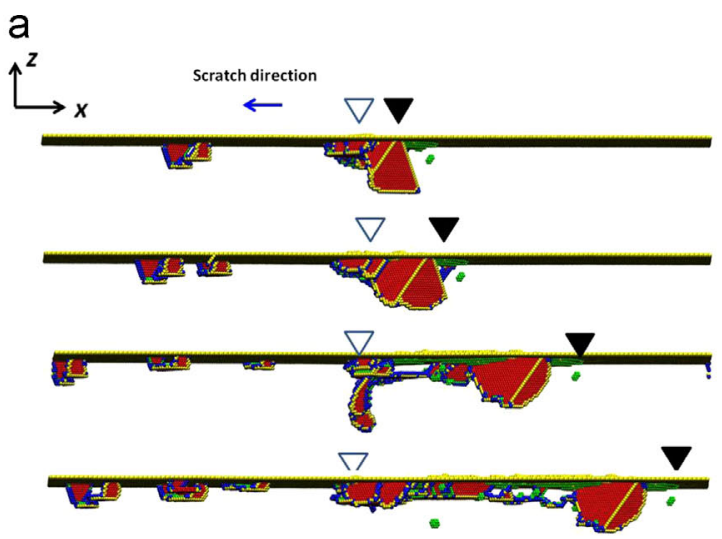

b
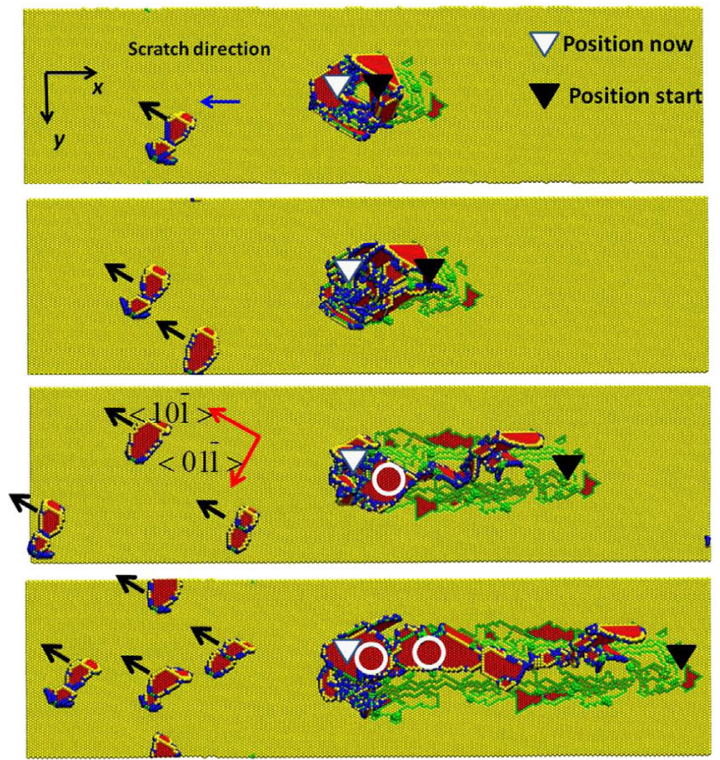

C
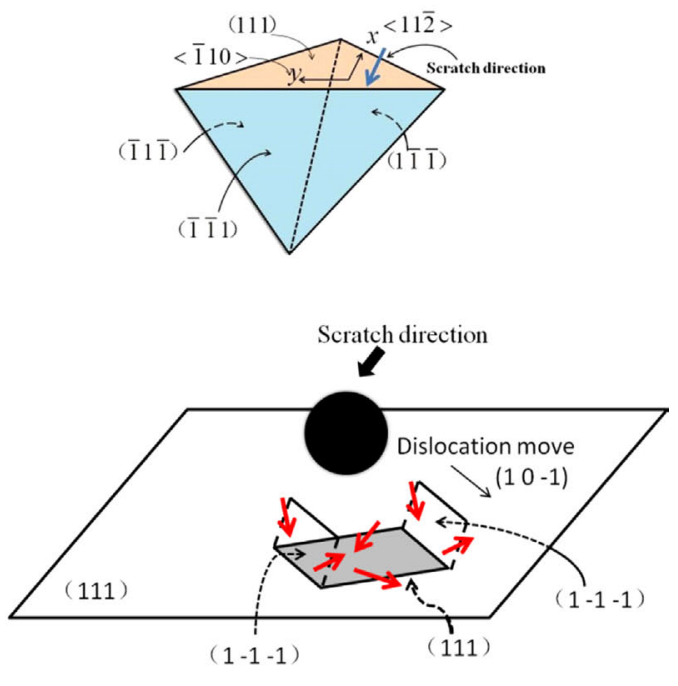

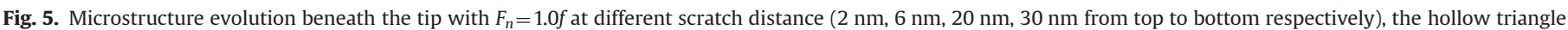

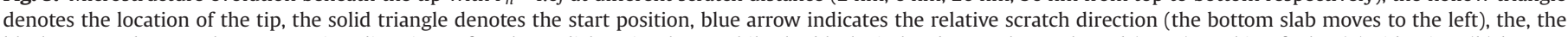

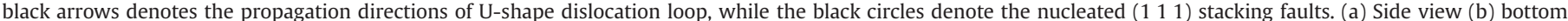

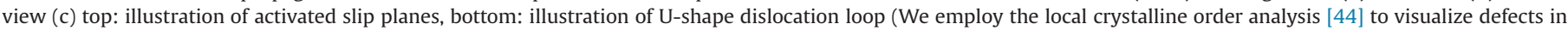

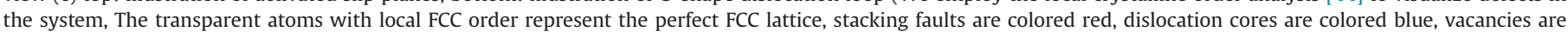

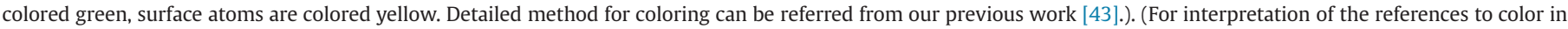
this figure legend, the reader is referred to the web version of this article.)

normal force on all the slip systems of ( $\left.\begin{array}{lll}1 & 1 & 1\end{array}\right)$ plane are zero, so the stacking faults on slip plane ( $\left.\begin{array}{lll}1 & 1 & 1\end{array}\right)$ are not activated in this stage.

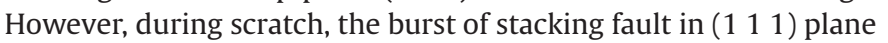

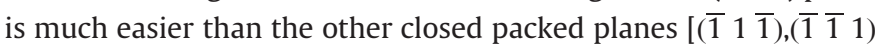

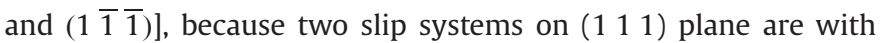
pretty high Schmid factor (0.866) with shear load. So, the initiation

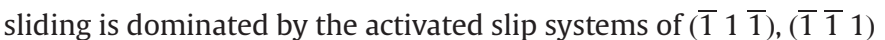
and $(1 \overline{1} \overline{1})$ planes extending to compensate the increase of scratch force. The decrease of the scratch force in stable sliding stage is caused by the activation of stacking faults on (1 111 ) slip plane. Also, as illustrated in Fig. $5 \mathrm{c}$ and the Schmid factor Table 1, Schmid

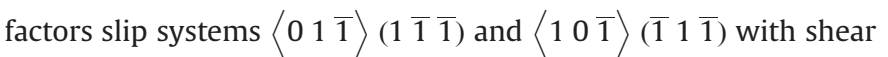
load are of the value of 0.289 , thus the U-shape dislocation loop are preferred to emit from the front of the tip as shown in Figs. 5 and 6. Each face of the U-shape dislocation loop consists of one full dislocation (a ribbon of stacking faults bounded by two partial dislocations) on (1 111 ) slip plane and two full dislocations on either $\left(\begin{array}{lll}1 & \overline{1} & \overline{1}\end{array}\right)$ or $\left(\begin{array}{ll}1 & 1 \\ 1\end{array}\right)$. U-shape dislocation loop propagate in the directions of either $\left\langle\begin{array}{lll}1 & 0 & \overline{1}\end{array}\right\rangle$ or $\left\langle\begin{array}{lll}0 & 1 & \overline{1}\end{array}\right\rangle$, as marked by black arrows in Figs. 5 and 6. This phenomenon also reduces the stress concentration in front the tip, thus leads a low value of friction force.

The burst of stacking faults hold the key for the plastic deformation of nano-scale material [39,40], in our study, the number of HCP (hexagonal close packed) atoms is recorded as a function of scratch distance, as shown in Fig. 7, scratch under higher normal force produces larger number of stacking faults as a result of larger plastic area beneath the tip. The force dependence of HCP number is shown in Fig. 8, the number of stacking faults atoms is more than doubled during the initiation of sliding, and as discussed above, at the initiation stage, most of them are activated

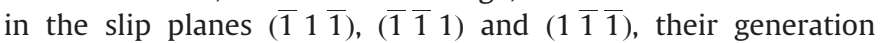
requires much higher friction force than in the slip plane of $\left(\begin{array}{lll}1 & 1 & 1\end{array}\right)$. The point where friction coefficient is in the stable regime corresponds to the moment for nucleation of stacking faults on (1 111 ) slip plane. The generation of the stacking faults on (1 111 ) plane is the cause of the transition of $\mu_{s}$ to $\mu_{d}$.

In continuum theory, a load dependent friction model for fully plastic condition is proposed in [41], which shows that the coefficient of friction decreases with increasing nominal contact 


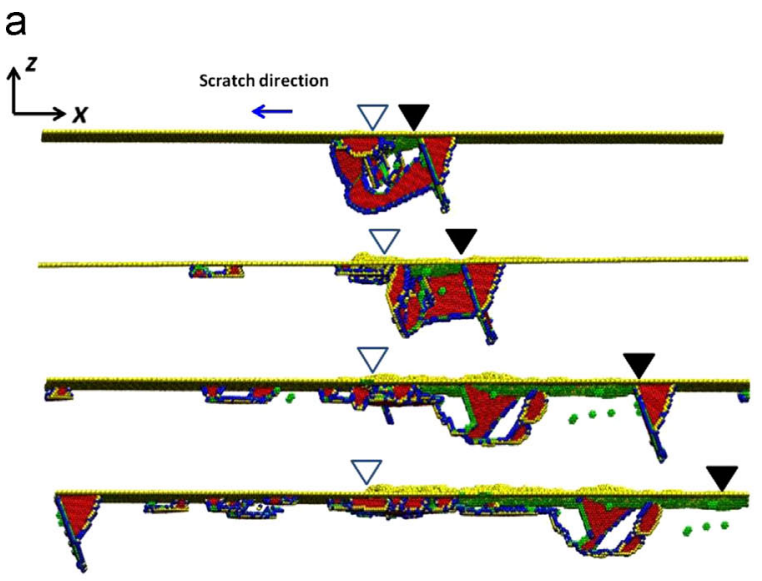

b
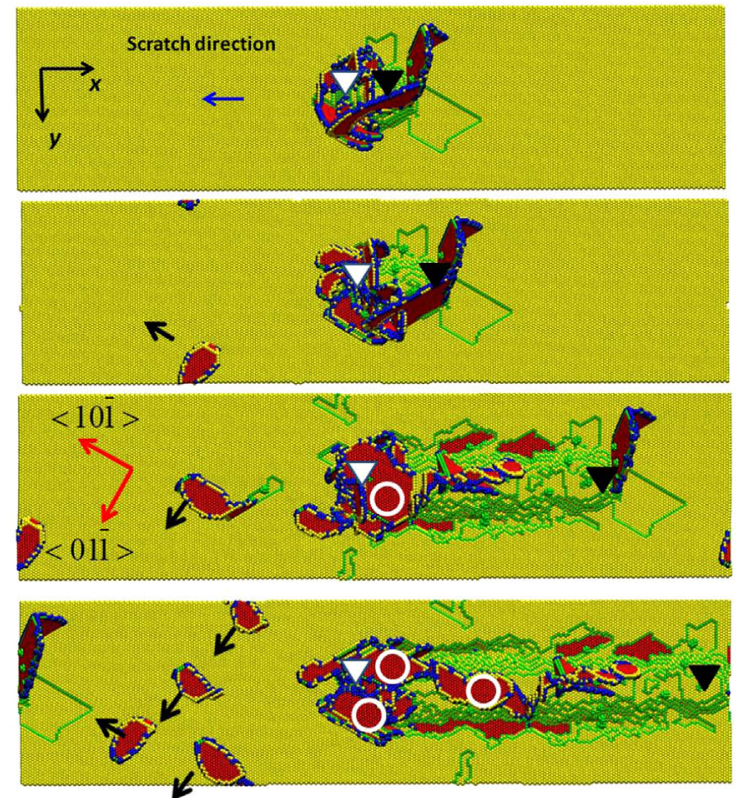

Fig. 6. Microstructure evolution beneath the tip with $F_{n}=1.2 f$ at different scratch distance ( $2 \mathrm{~nm}, 6 \mathrm{~nm}, 20 \mathrm{~nm}, 32 \mathrm{~nm}$ from top to bottom, respectively). (a) Side view (b) bottom view.

Table 1

Schmid factor on 12 slip systems for normal load and shear load.

\begin{tabular}{|c|c|c|c|}
\hline \multirow[t]{2}{*}{ Slip plane } & \multirow[t]{2}{*}{ Slip direction } & \multicolumn{2}{|l|}{ Schmid factor } \\
\hline & & Normal load & Shear load \\
\hline \multirow[t]{3}{*}{$\left(\begin{array}{lll}1 & 1 & 1\end{array}\right)$} & $\langle 0 \overline{1} 1\rangle$ & 0 & 0.866 \\
\hline & $\left\langle\begin{array}{lll}\overline{1} & 0 & 1\end{array}\right\rangle$ & 0 & 0.866 \\
\hline & $\langle\overline{1} 110\rangle$ & 0 & 0 \\
\hline \multirow[t]{3}{*}{$(\overline{1} \overline{1} 1)$} & $\left\langle\begin{array}{lll}0 & 1 & 1\end{array}\right.$ & 0.272 & 0.096 \\
\hline & $\left\langle\begin{array}{lll}1 & 0 & 1\end{array}\right\rangle$ & 0.272 & 0.096 \\
\hline & $\langle 1 \overline{1} 0\rangle$ & 0 & 0 \\
\hline \multirow[t]{3}{*}{$(1 \overline{1} \overline{1})$} & $\left\langle\begin{array}{lll}0 & 1 & \overline{1}\end{array}\right.$ & 0 & 0.289 \\
\hline & $\langle\overline{1} \quad 0 \overline{1}\rangle$ & 0.272 & 0.096 \\
\hline & $\left\langle\begin{array}{lll}\overline{1} & \overline{1} & 0\end{array}\right.$ & 0.272 & 0.193 \\
\hline \multirow[t]{3}{*}{$(\overline{1} 1 \overline{1})$} & $\langle 0 \overline{1} \overline{1}\rangle$ & 0.272 & 0.096 \\
\hline & $\left\langle\begin{array}{lll}1 & 0 & \overline{1}\end{array}\right.$ & 0 & 0.289 \\
\hline & $\left\langle\begin{array}{lll}1 & 1 & 0\end{array}\right\rangle$ & 0.272 & 0.193 \\
\hline
\end{tabular}

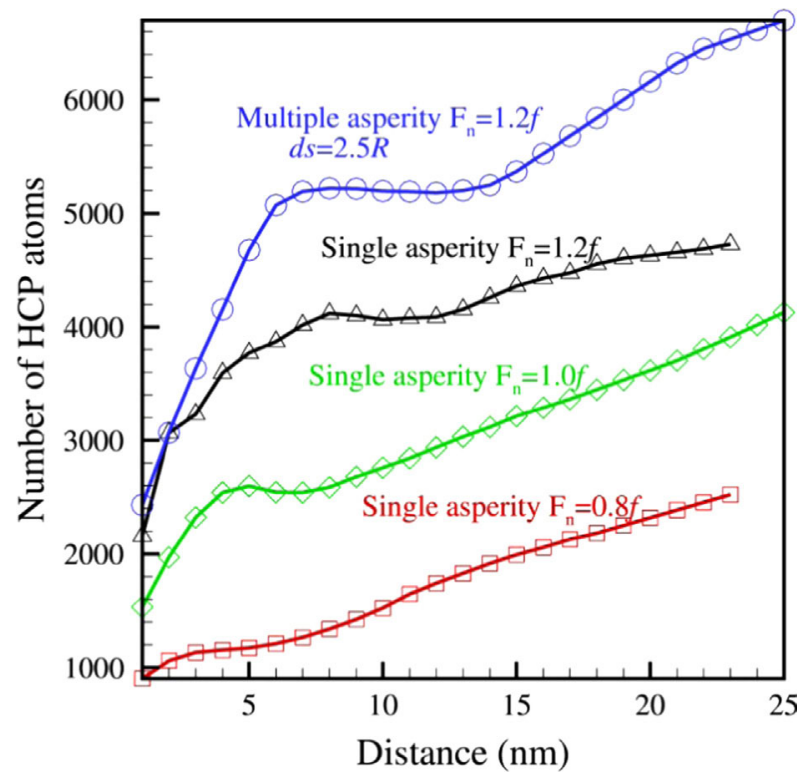

Fig. 7. Number of HCP atoms generated per asperity as a function of scratch distance, the results are averaged over ten cases for single asperity, and sixteen for asperity array.

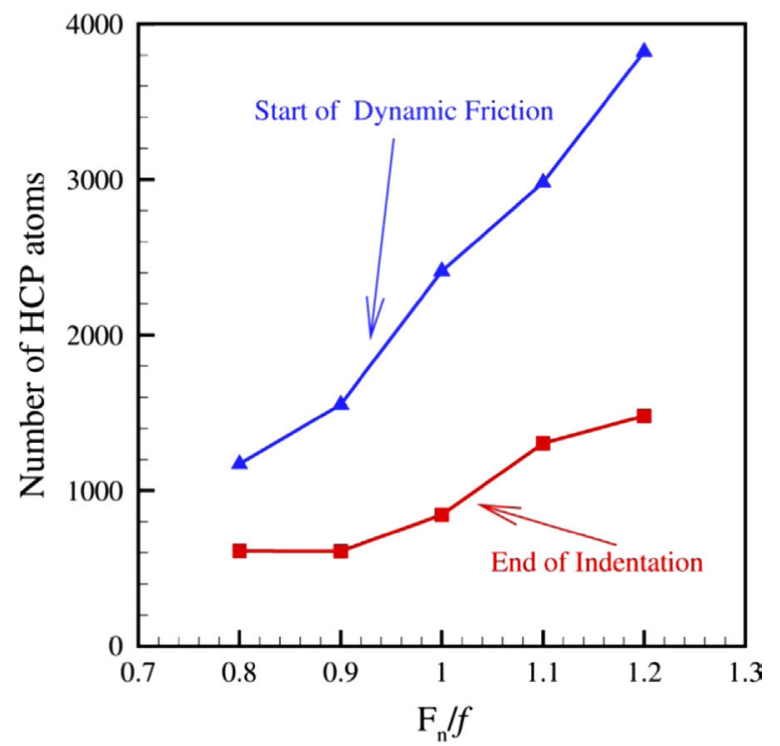

Fig. 8. Burst of stacking fault during the initiation of sliding (all the data points are averaged over ten cases).

pressure. While in nano-friction, our study shows the opposite (see in Fig. 4b). The major reason is that the evolution of discrete dislocation and stacking fault play a key role during nano-scratch. The tip with higher normal force produces larger number of stacking faults (see in Fig. 7), which generate higher value of friction force. And also the pile-up of atoms in front of the tip is another crucial factor [7], which will be discussed later.

\subsection{Interference effect of asperity array}

The studies on hardness of nano-scale metallic contacts [12,13] show that little interaction between nearly asperities is observed during indentation, however, the interference effect between asperities in scratch test is rather big compared within indentation test. Single asperity friction has an obvious difference between static friction and dynamic friction, while it is not the case for the multi-asperity friction, as seen in Fig. 9 for the case of $d s=2 R$, none 


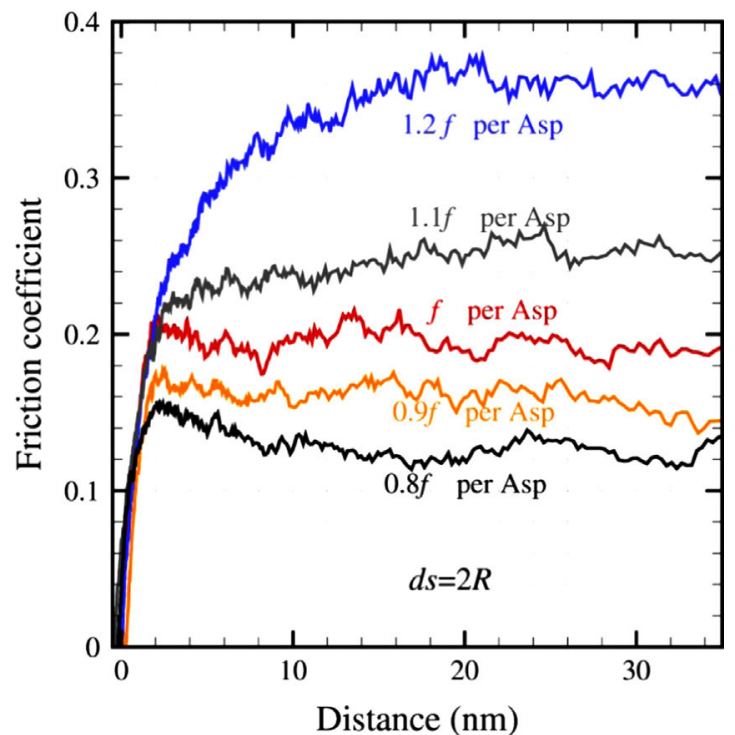

Fig. 9. Friction curves of asperity array with inter distance $(d s=2 R)$.

of the friction curves show a abrupt decrease of friction coefficient as the initiation of sliding. In order to study the mechanism of smooth transition of the friction curve, the dislocation patterns during the sliding are shown in Figs. 10 and 11 (Without losing universality, we show the cases with $d s=2 R, \theta=0^{\circ}$ and $d s=2.5 R$, $\theta=45^{\circ}$, respectively). For multi-asperities, the stacking fault patterns don't show a big difference between the initiation stage of sliding and stable sliding stage, the stacking faults are always on

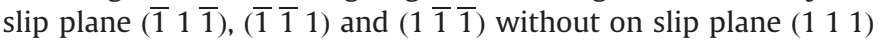
which requires the lower sliding force. The patterns are large area of intersections between the staking faults generated by near asperities, as shown in Figs. 10 and 11. The intersections make a complex microstructure so that the stacking faults on slip plane (1 11 1) can't be activated [13].

Not like single asperity sliding (see in Figs. 5 and 6), few Ushape dislocation loops are observed for the case of multiasperities. The interactions of partial dislocation and stacking fault beneath asperities prevent the propagation of U-shape dislocation loop and also the formation of stacking faults on (1 111 ) slip plane. After scratch over about $20 \mathrm{~nm}$, the friction curve comes into the stable sliding stage, as shown in Fig. 12.

The normal load effect on friction transition is shown in Fig. 12. While $F_{n}$ is small with the value $0.8 f$, the interference effect is trivial, all the friction curves have a similar pattern. It has a maximum point denoting static friction coefficient, an abrupt drop and a stable sliding stage afterwards (see in Fig. 12a). While $F_{n}$ is large with the value $1.2 f$, the maximum static friction can be observed for single asperity, the static-dynamic transition is obvious, the friction transition goes to smooth as the inter distance decreases for multi-asperities. It can be concluded that: For single asperity contact, initiation sliding always has to conquer the maximum static resistance; For the case of multi-asperities, static-dynamic friction transition is only expected when the normal force is small (each asperity is almost independent).

Spijker et al. [42] showed that surface roughness is the dominant parameter for the sliding of deformable solids, the friction is reduced to zero as the surface is flattened. In our case, the asperities represent rough surface of cutting tool, they are considered as rigid compared to substrate. So, the plowing effect is studied in this work, rather than surface flattening effect in atomistic friction research $[11,42]$. The averaged friction coefficient for multi-asperities is summarized in Fig. 13. For single asperity, the normal load dependence of friction coefficient is almost linear, a
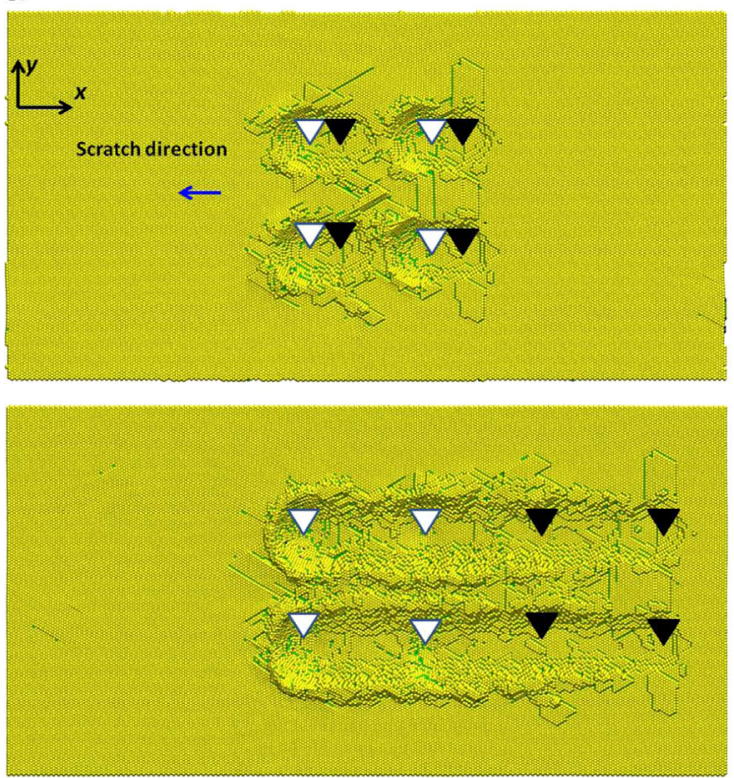

b
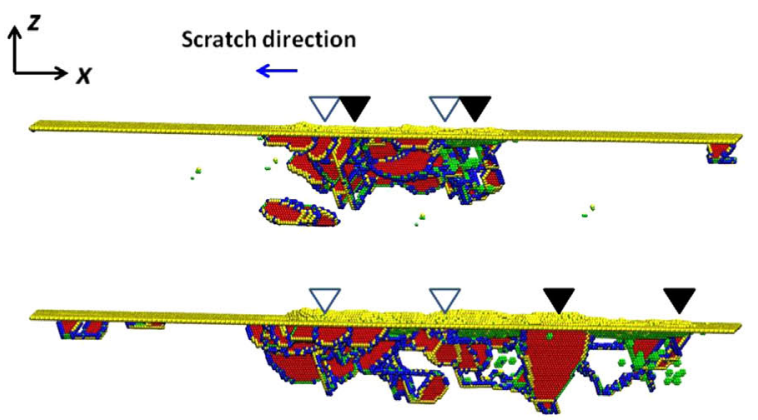

C
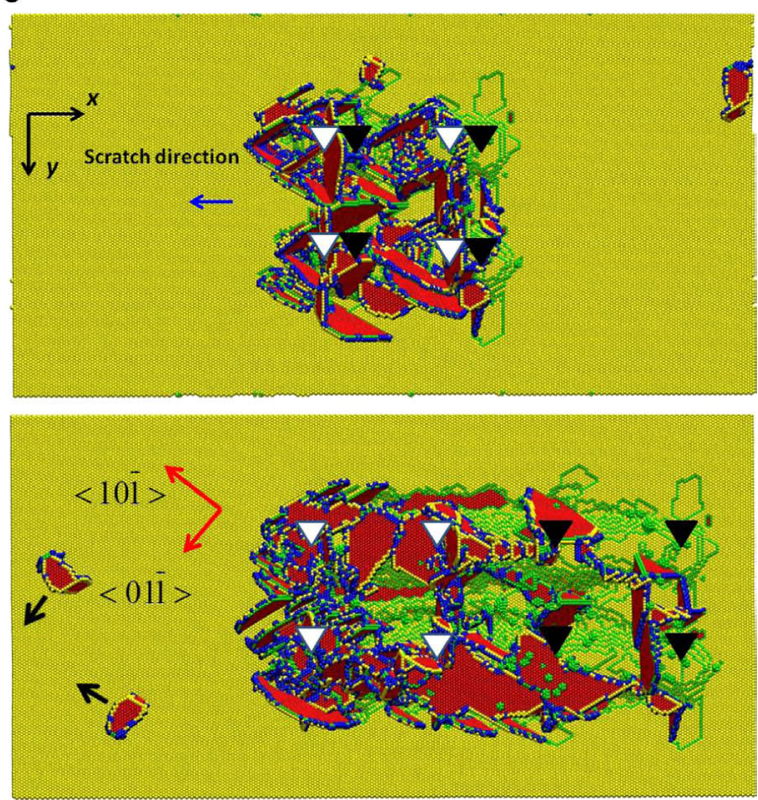

Fig. 10. Microstructure evolution of asperity array $\left(\theta=0^{\circ}, d s=2.5 R\right)$ beneath the tip with $F_{n}=1.2 f$ per asperity at different scratch distance $(6 \mathrm{~nm}, 20 \mathrm{~nm}$ from top to bottom, respectively) (a) top view (b) side view (c) bottom view.

which agrees with the previous studies $[3,43]$ with non-adhesive sliding. As the inter-distance $d s$ decreases, the effect of normal load becomes significant. The value of $\mu_{d}$ increases around $78 \%$ as 
a
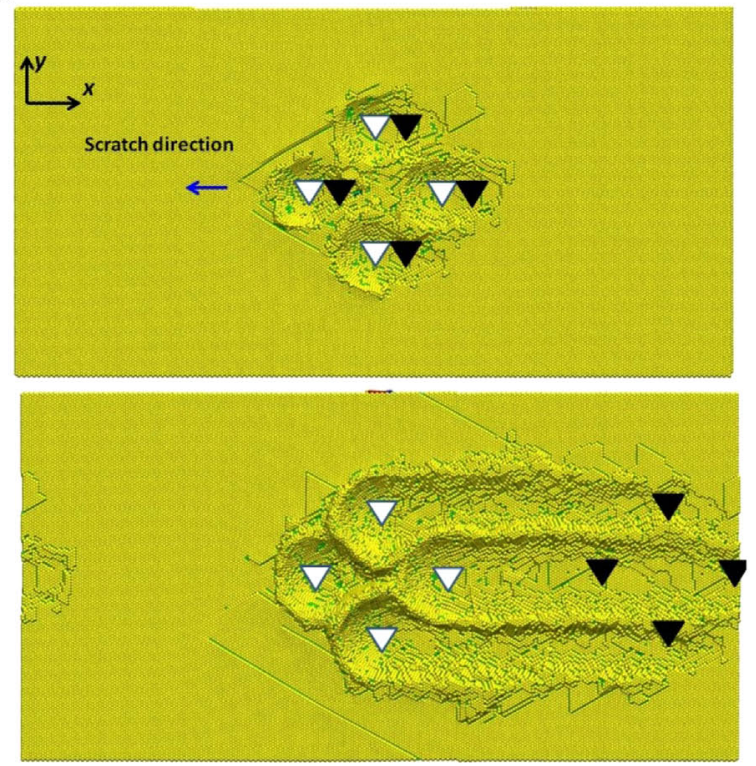

b

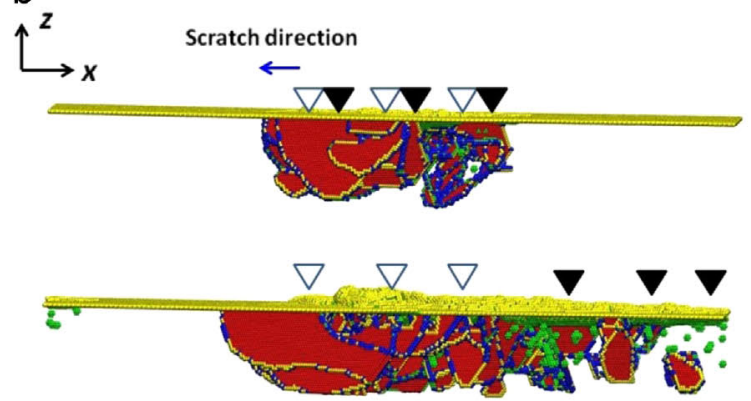

C
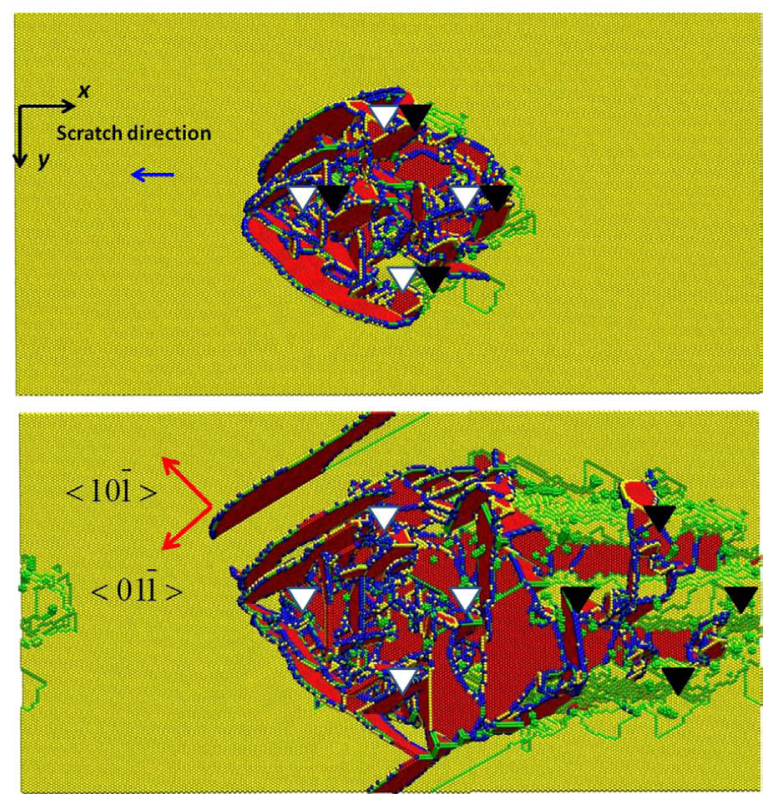

Fig. 11. Microstructure evolution of asperity array $\left(\theta=45^{\circ}, d s=2.5 R\right)$ beneath the tip with $F_{n}=1.2 f$ per asperity at different scratch distance $(6 \mathrm{~nm}, 25 \mathrm{~nm}$ from top to bottom, respectively) (a) top view (b) side view (c) bottom view.

$F_{n}$ increases from $0.8 f$ to $1.2 f$ for single asperity, While the value of $\mu_{d}$ increases around $188 \%$ as $F_{n}$ increases from $0.8 f$ to $1.2 f$ for multiple asperity with $d s=2 R$. The mechanism for the increase of $\mu_{d}$ are: First is the generation of more stacking faults in case of a

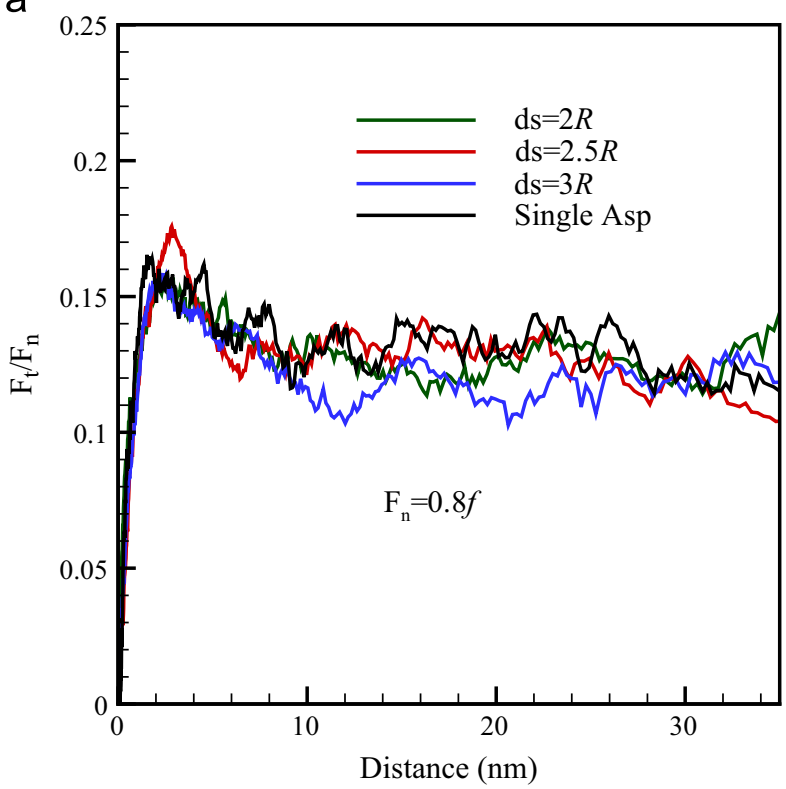

b

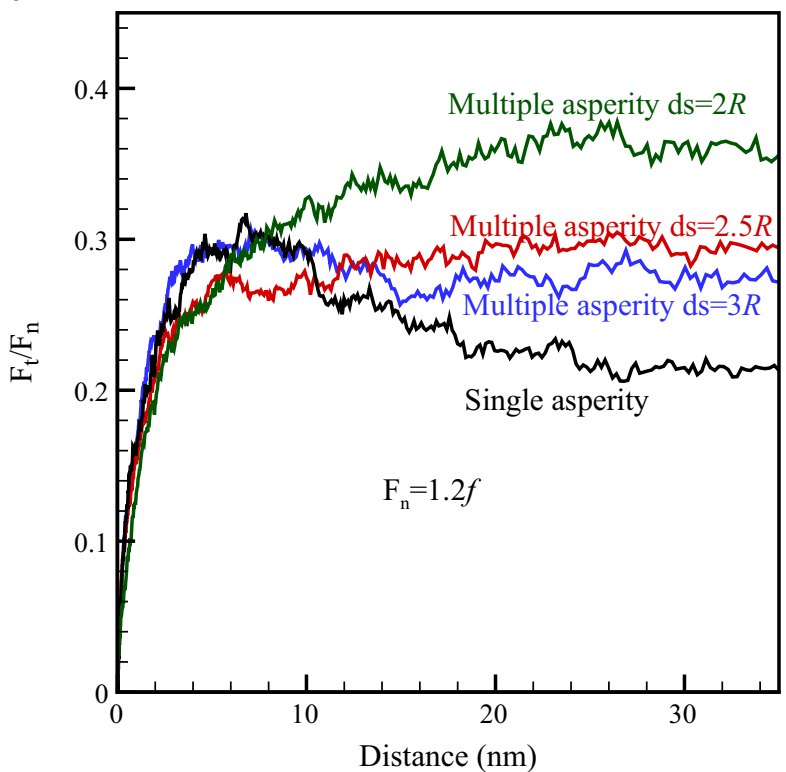

Fig. 12. comparison of friction behavior between single asperity and multiple asperity (a) low value of normal load $0.8 f$ per asperity; (b) high value of normal load $1.2 f$ per asperity.

multi-asperities, as it can be seen in Fig. 7, it generates more stacking faults as a result of asperity interference; Second is the emission of U-shape dislocation loop. In single asperity case, without interference, U-shape dislocation loops are emitted to release the stress concentration in front of the tip, the dislocation emission reduces the friction force. The interference of multiasperities prevents the emission of U-shape dislocation loop, this may also cause high local stress. Third is the pile-up of atoms in front the asperities. The number of pile-up atoms is compared in Fig. 14, pile-up of atoms increases with the increase of normal load, it also increases as the $d s$ decreases. The pile-up volume is almost the same for low normal load $\left(F_{n}=0.8 f, 0.9 f\right)$, which is also reflected in the $\mu_{d}-F_{n}$ relation in Fig. 12. The pile-up volume with multi-asperity ( $d s=2 R F_{n}=1.2 f$ ) is almost twice compared with the single asperity case with the same normal load. The detailed geometry after scratch of $25 \mathrm{~nm}$ is shown in Fig. 15 for comparison. 


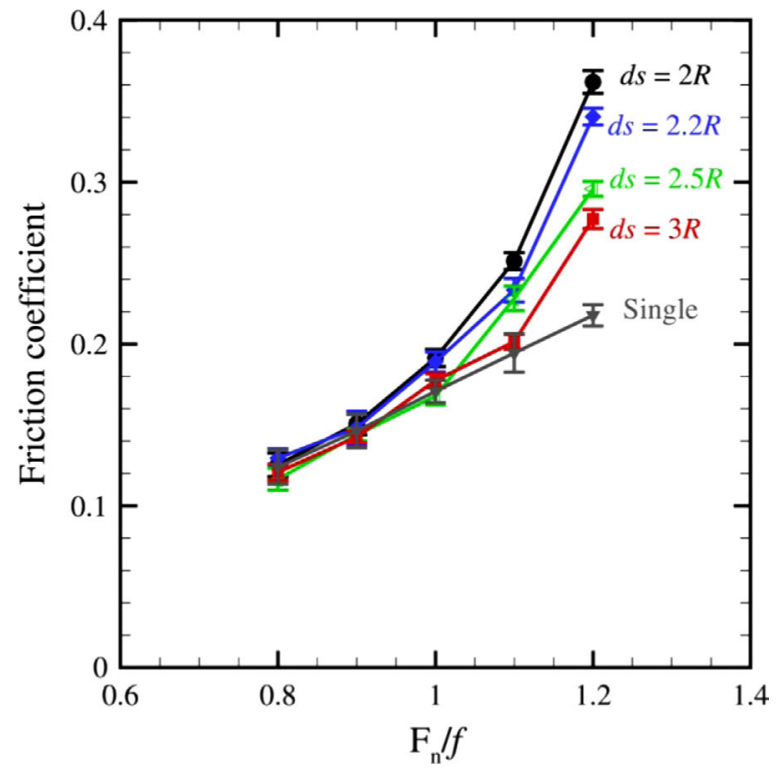

Fig. 13. Change of friction coefficient with different interference values of the asperity array, error bars denote the standard deviation from the scratch distance over $25 \mathrm{~nm}$ to $35 \mathrm{~nm}$.

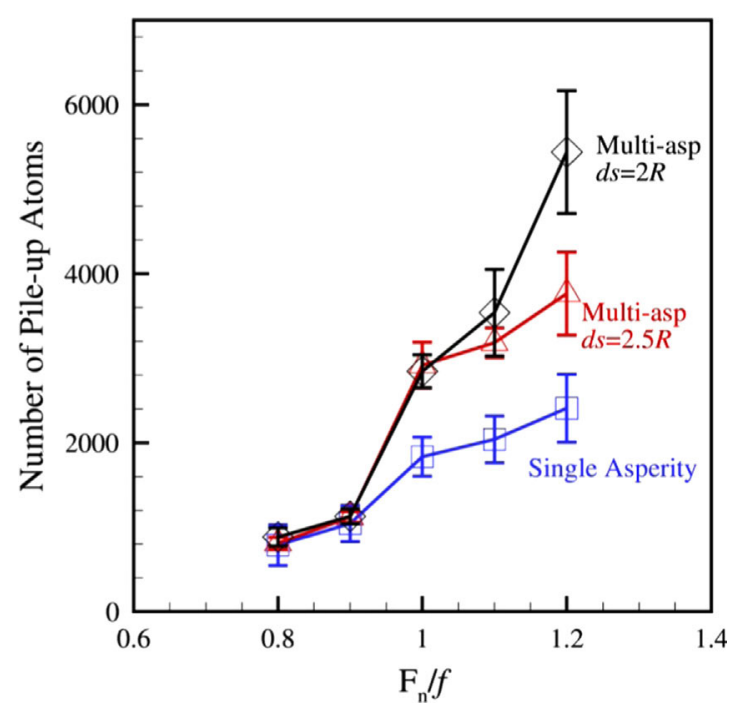

Fig. 14. Number of pile-up atoms per asperity after scratch of $25 \mathrm{~nm}$, error bar denotes standard deviation over cases. (The atoms which are above the surface are considered as pile-up, the reference of surface is defined by the surface atoms far away from the scratch point.).

\section{Conclusion}

The friction behaviors of asperity and multi-asperities on (1 111 1) surface of single crystal copper are studied by using Green function molecular dynamics method, conclusions are as follows:

1. Single asperity scratch shows a clear transition from $\mu_{s}$ to $\mu_{d}$ : Change of the activation slip systems is the main reason of the friction coefficient transition. Generation of (1 111 ) stacking faults (parallel to the surface) requires smaller value of friction force. The larger value of static friction comes from the burst of stacking faults at the initiation stage of sliding.

2. The $\mu_{s}-\mu_{d}$ transition disappears for scratch of multi-asperities with high normal load, the interactions of stacking faults a

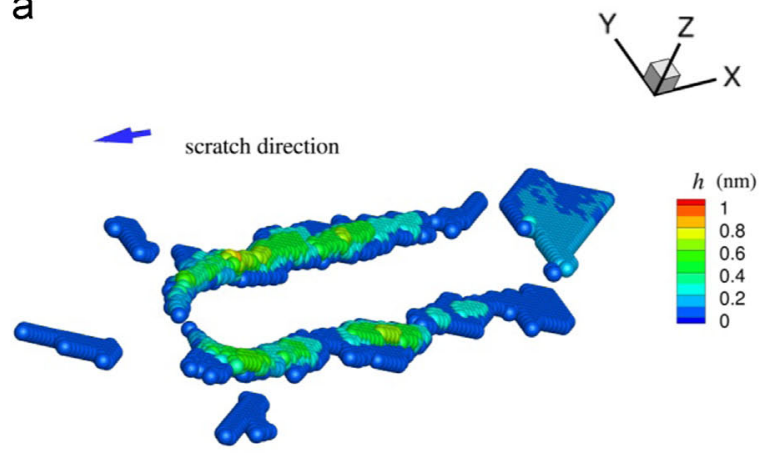

b
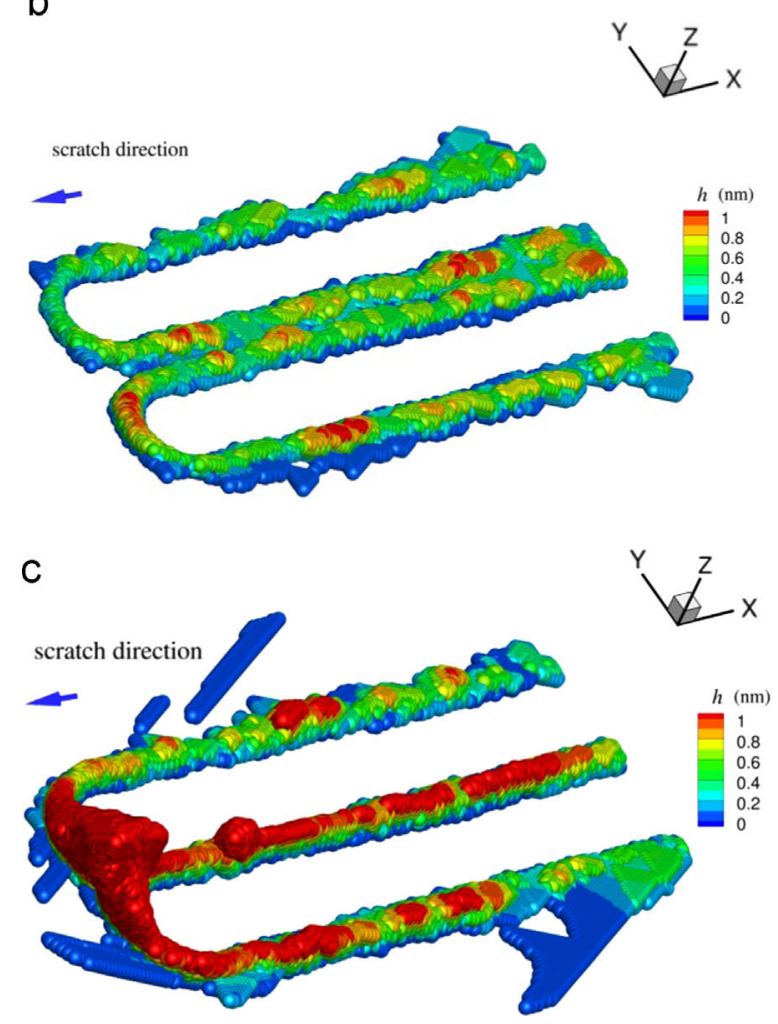

Fig. 15. Pile-up patterns after scratch of $25 \mathrm{~nm}$ with $F_{n}=1.2 f$ per asperity (a) Single asperity (b) asperity array with $\theta=0^{\circ} d s=2.5 R$ (c) asperity array with $\theta=0^{\circ}$, $d s=2.0 R$. The $h$ indicates the pile-up height, the reference of surface is defined by the surface atoms far away from the scratch point.

beneath asperities prevent generation of easily sliding plane (1 111 ) and hinder the propagation of U-shape dislocation loop. 3. Nearly linear load dependence of friction coefficient is observed for single asperity. The load dependence is dramatically increased for friction of multi-asperities. Atomistic pile-up is one reason for the increase of friction coefficient with multiasperities. The strengthening interaction of stacking faults resulting from interference also contributes to the change of friction coefficient.

\section{Acknowledgements}

This work was supported by National Basic Research Program of China through 2012CB937500, and by the National Natural Science Foundation of China (Grant no.11202214, no. 91216108, no. 11372131), by State Key Laboratory of Mechanics and Control of 
Mechanical Structures(NUAA Grant no. MCMS-0114G01). Insightful discussions about applying Green function method with Prof. M.H. Müser and Prof. L.T. Kong are gratefully acknowledged. Authors thank Prof. Yujie Wei for useful comments.

\section{References}

[1] Johnson KL. Contact mechanics. Cambridge university press; 1987.

[2] Derjaguin B, Muller V, Toporov YP. Effect of contact deformations on the adhesion of particles. J Colloid Interface Sci 1975;53(2):314-26.

[3] Mo YF, Turner KT, Szlufarska I. Friction laws at the nanoscale. Nature 2009;457 (7233):1116-9.

[4] Mo YF, Szlufarska I. Roughness picture of friction in dry nanoscale contacts. Phys Rev B: Condens Matter 2010;81:3.

[5] Wang DF, Kato K. Effect of coating thickness on friction for carbon nitride films in repeated sliding against a spherical diamond with nano-scale asperities. Wear 2002;252(3-4):210-9.

[6] Yang LQ, Martini A. Nano-scale roughness effects on hysteresis in micro-scale adhesive contact. Tribol Int 2013;58:40-6.

[7] Mishra M, Szlufarska I. Analytical model for plowing friction at nanoscale. Tribol Lett 2012:45(3):417-26.

[8] Mishra M, et al. Friction model for single-asperity elastic-plastic contacts. Phys Rev B: Condens Matter 2012;86:4

[9] Tartaglino U, Samoilov VN, Persson BN. Role of surface roughness in superlubricity. J Phys Condens Matter 2006;18(17):4143.

[10] Yang L, Martini A. Nano-scale roughness effects on hysteresis in micro-scale adhesive contact. Tribol Int 2013;58:40-6.

[11] Spijker P, Anciaux G, Molinari J-F. Dry sliding contact between rough surfaces at the atomistic scale. Tribol Lett 2011;44(2):279-85.

[12] Kim H, Strachan A. Nanoscale metal-metal contact physics from molecular dynamics: the strongest contact size. Phys Rev Lett 2010;104(21):215504.

[13] Kim H, Strachan A. Size-dependent hardness of nanoscale metallic contacts from molecular dynamics simulations. Phys Rev B: Condens Matter 2012;86 (6):064101.

[14] Greer JR, Oliver WC, Nix WD. Size dependence of mechanical properties of gold at the micron scale in the absence of strain gradients. Acta Mater 2005;53 (6): $1821-30$.

[15] Shan Z, et al. Mechanical annealing and source-limited deformation in submicrometre-diameter Ni crystals. Nat Mater 2007;7(2):115-9.

[16] Pastewka L, Sharp TA, Robbins MO. Seamless elastic boundaries for atomistic calculations. Phys Rev B: Condens Matter 2012;86(7):075459.

[17] Kong LT, et al. Implementation of Green's function molecular dynamics: an extension to LAMMPS. Comput Phys Commun 2009;180(6):1004-10.

[18] Kong LT. Phonon dispersion measured directly from molecular dynamics simulations. Comput Phys Commun 2011;182(10):2201-7.

[19] Campaná C, Müser MH. Contact mechanics of real vs. randomly rough surfaces: a Green's function molecular dynamics study. EPL (Eur. Lett.) 2007;77(3):38005

[20] Zhang Q, et al. Origin of static friction and its relationship to adhesion at the atomic scale. Phys Rev B: Condens Matter 2007;75(14):144114.

[21] Zhang Q et al. Origin of static friction and its relationship to adhesion at the atomic scale. Phys Rev B: Condens Matter 2007;75:14.
[22] Junge T, Molinari J-F. Plastic activity in nanoscratch molecular dynamics simulations of pure aluminium. Int J Plast 2014;53:90-106.

[23] Komanduri R, Raff L. A review on the molecular dynamics simulation of machining at the atomic scale. Proc Inst Mech Eng Part B J Eng Manuf 2001;215(12):1639-72.

[24] Hasnaoui A, Derlet P, Van Swygenhoven H. Interaction between dislocations and grain boundaries under an indenter-a molecular dynamics simulation. Acta Mater 2004;52(8):2251-8.

[25] Zhang J, et al. Twin boundary spacing-dependent friction in nanotwinned copper. Phys Rev B: Condens Matter 2012;85(5):054109.

[26] Si L, et al. Monoatomic layer removal mechanism in chemical mechanical polishing process: a molecular dynamics study. J Appl Phys 2010;107 (6):064310.

[27] Kelchner CL, Plimpton S, Hamilton J. Dislocation nucleation and defect structure during surface indentation. Phys Rev B: Condens Matter 1998;58 (17): 11085.

[28] Li J, et al. Atomistic mechanisms governing elastic limit and incipient plasticity in crystals. Nature 2002;418(6895):307-10.

[29] Feichtinger D, Derlet P, Van Swygenhoven H. Atomistic simulations of spherical indentations in nanocrystalline gold. Phys Rev B: Condens Matter 2003;67(2):024113.

[30] Jun S, et al. Large-scale molecular dynamics simulations of Al (1 111$)$ nanoscratching. Nanotechnology 2004;15(9):1169.

[31] Hu Y-z, Ma T-b, Wang H. Energy dissipation in atomic-scale friction. Friction 2013;1(1):24-40.

[32] Filleter T, Bennewitz R. Nanometre-scale plasticity of Cu (100). Nanotechnology 2007;18(4):044004.

[33] Asenjo A, et al. Dislocation mechanisms in the first stage of plasticity of nanoindented Au (1 11 ) surfaces. Phys Rev B: Condens Matter 2006;73 (7):075431.

[34] Bhushan B. Nanotribology and nanomechanics. Wear 2005;259(7):1507-31.

[35] Li Q et al. Speed dependence of atomic stick-slip friction in optimally matched experiments and molecular dynamics simulations. Phys Rev Let 2011;106(12):126101.

[36] Gotsmann B, Lantz MA. Atomistic wear in a single asperity sliding contact. Phys Rev Lett 2008;101(12):125501.

[37] Si L, et al. Abrasive rolling effects on material removal and surface finish in chemical mechanical polishing analyzed by molecular dynamics simulation. J Appl Phys 2011;109(8):084335.

[38] Si L, et al. Planarization process of single crystalline silicon asperity under abrasive rolling effect studied by molecular dynamics simulation. Appl Phys A 2012;109(1):119-26.

[39] Ma X-L, Yang W. Molecular dynamics simulation on burst and arrest of stacking faults in nanocrystalline $\mathrm{Cu}$ under nanoindentation. Nanotechnology 2003;14(11):1208.

[40] Mordehai D, Rabkin E, Srolovitz DJ. Pseudoelastic deformation during nanoscale adhesive contact formation. Phys Rev Lett 2011;107(9) (096101-096101).

[41] Ma XA, de Rooij M, Schipper D. A load dependent friction model for fully plastic contact conditions. Wear 2010;269(11-12):790-6.

[42] Spijker P, Anciaux G, Molinari J-F. Relations between roughness, temperature and dry sliding friction at the atomic scale. Tribol Int 2013;59:222-9.

[43] Liu X, Liu Z, Wei Y. Nanoscale friction behavior of the Ni-film/substrate system under scratching using MD simulation. Tribol Lett 2012;46(2):167-78.

[44] Honeycutt JD, Andersen HC. Molecular dynamics study of melting and freezing of small Lennard-Jones clusters. J Phys Chem 1987:91(19):4950-63. 